\title{
An astrometric catalogue for the area of Coma Berenices
}

\author{
C. $\mathrm{Abad}^{1}$ and B. Vicente ${ }^{2}$ \\ 1 Centro de Investigaciones de Astronomía CIDA, 5101-A Mérida, Venezuela \\ ${ }^{2}$ Grupo de Mecánica Espacial, Departamento de Física Teórica, Universidad de Zaragoza, 50006 Zaragoza, Spain
}

Received September 25, 1998; accepted January 14, 1999

\begin{abstract}
A catalogue ${ }^{1}$ of stellar positions and proper motions down to the 14th photographic magnitude in the area of the open cluster in Coma Berenices is compiled from data of 12 different sources. The accuracy of the proper motion data is comparable to that of the Hipparcos Catalogue.
\end{abstract}

Key words: astrometry — catalogues — methods: data analysis — open clusters: coma cluster

\section{Introduction}

The principal purpose of this work is to present a catalog of positions and proper motions for stars in the area of the open star cluster in Coma Berenices. The data are based on a compilation of 12 different catalogs. The first exhaustive study of the cluster was carried out by Trumpler (1938), determining definite cluster membership for the brighter stars. For some of the fainter stars the membership remained in doubt. The small number of members, a little more than 50 , can be considered to be characteristic for most of the known open clusters. The determination of membership probability based on proper motions is somewhat difficult in this case because the motion of the cluster is not very different from that of the surrounding field stars. Thus it is important to have proper motions of high accuracy for the selection of cluster members which is the aim of this work. The precision of the proper motions determined in this paper is comparable to that of the Hipparcos data. This is due to the large epoch difference covered by the catalogs which contribute to the compilation of data, to the quality of the plate model used in the reduction of a series of photographic plates,

Send offprint requests to: C. Abad

1 The catalogue Table 5 is only available in electronic form at the CDS via anonymous ftp to cdsarc.u-strasbg.fr (130.79.128.5) or via http://cdsweb.ustrasbg.fr/Abstract.html and to the validity of the process with which systematic errors with respect to the Hipparcos system were eliminated from the various source data. Initially this work was part of the Ph. D. thesis of C. Abad (Abad 1996), where the PPM Catalogue of Positions and Proper Motions by Bastian \& Roeser (1991) was used as reference system. The Hipparcos Catalogue was not available yet at that time. The reduction method employed was that described by Abad (1993). In this work, the reference system and the reduction method were both improved. The reference system is now the Hipparcos Catalogue, and the reduction is based on a model described by Abad (1998). The catalog compiled in this paper is based on 12 source catalogs of different types. They include meridian circle observations, photographic plates, and position observations with CCD detectors. One of the sources is photographic plates obtained with the CIDA 1-meter Schmidt telescope, and it is these plates which define the limits of the area survey. The source catalogs for which we either had the plates or the measured rectangular coordinates $X, Y$ of stellar objects were reduced in the process. We wish to mention in particular the POSS plates whose microdensitometer scans were made available to us by the Space Telescope Science Institute. A list of all sources, giving the respective epoch and indicating whether the date was reduced by us or not is given in Table 1 .

\section{Reduction of the photographic plates}

The process of the reduction of photographic plates starts with the determination of the cartesian coordinates $X$ and $Y$. For the catalogs POSS, Schmidt 88, and Schmidt 94 the plates were first digitized with PDS microdensitometers. The plates of the series Schmidt 88 were digitized with the PDS microdensitometer of the Observatory of Munster, Germany, and the coordinates $X$ and $Y$ were determined using their computer software. The plates of the series Schmidt 94 were digitized with the PDS microdensitometer of the Department of Astronomy of the Yale University. The respective $X$ and $Y$ coordinates were 
Table 1. List of used catalogues

\begin{tabular}{|r|c|r|r|c|c|}
\hline Number & Mean epoch & Catalogue & Type & Reduction & Numbre of stars \\
\hline 1 & 1900 & Paris & photographic & yes & 675 \\
2 & 1900 & Oxford & photographic & yes & 2783 \\
3 & 1926 & Heckmann & photographic & yes & 212 \\
4 & 1928 & Yale & meridian & no & 280 \\
5 & 1930 & AGK2 & photographic & no & 383 \\
6 & 1956 & POSS & photographic & yes & 8930 \\
7 & 1958 & AGK3 & photographic & no & 383 \\
8 & 1988 & Schmidt88 CIDA & photographic & yes & 3359 \\
9 & 1990 & Schmidt90 CIDA & photographic & yes & 4951 \\
10 & 1991 & Carlsberg & meridian & no & 301 \\
11 & 1991 & Tycho & Hipparcos & no & 702 \\
12 & 1994 & Schmidt94 CIDA & photographic & yes & 5924 \\
\hline \hline
\end{tabular}

Table 2. Final errors for the entire Paris Zone and part of the Oxford Zone of the AC and for the POSS plates covering the coma area

\begin{tabular}{|r|rrr|rrr|rr|r|}
\hline \multicolumn{1}{|c|}{ Errors } & \multicolumn{3}{|c|}{ AC Paris zone } & \multicolumn{3}{c|}{ AC Oxford zone } & \multicolumn{2}{|c|}{ POSS (PPM) } \\
\hline Magnitude & $\sigma_{\alpha}(\mathrm{s})$ & $\sigma_{\delta}\left({ }^{\prime \prime}\right)$ & img. & $\sigma_{\alpha}(\mathrm{s})$ & $\sigma_{\delta}\left({ }^{\prime \prime}\right)$ & img. & $\sigma_{\alpha}(\mathrm{s})$ & $\sigma_{\delta}\left({ }^{\prime \prime}\right)$ & img. | \\
\hline 2 & 0.014 & 0.34 & 6 & 0.039 & 0.47 & 10 & & & \\
3 & 0.029 & 0.54 & 17 & 0.023 & 0.52 & 10 & & & \\
4 & 0.019 & 0.34 & 79 & 0.034 & 0.32 & 68 & & & \\
5 & 0.018 & 0.23 & 331 & 0.023 & 0.31 & 79 & & & \\
6 & 0.016 & 0.21 & 1343 & 0.023 & 0.32 & 222 & 0.074 & 0.33 & 7 \\
7 & 0.014 & 0.20 & 4747 & 0.022 & 0.29 & 627 & 0.026 & 0.26 & 25 \\
8 & 0.014 & 0.20 & 14504 & 0.022 & 0.28 & 2058 & 0.028 & 0.40 & 56 \\
9 & 0.014 & 0.19 & 43720 & 0.022 & 0.28 & 6574 & 0.024 & 0.27 & 46 \\
10 & 0.013 & 0.19 & 114957 & 0.021 & 0.27 & 35301 & 0.020 & 0.26 & 168 \\
11 & 0.014 & 0.19 & 90985 & 0.022 & 0.29 & 36682 & 0.020 & 0.28 & 93 \\
12 & 0.015 & 0.20 & 7275 & 0.023 & 0.29 & 543 & 0.028 & 0.33 & 63 \\
13 & & & & & & & 0.018 & 0.41 & 18 \\
\hline total errors & 0.014 & 0.19 & 277974 & 0.022 & 0.28 & 82179 & 0.024 & 0.30 & $443 \mid$ \\
\hline *'s Hipp. & 0.018 & 0.25 & 6996 & 0.023 & 0.32 & 2398 & & & \\
\hline
\end{tabular}

Table 3. Final errors for the three Schmidt CIDA plates series covering the coma area

\begin{tabular}{|r|rrr|rrr|rrr|}
\hline \multicolumn{1}{|c|}{ Errors } & \multicolumn{2}{|c|}{ Schmidt plates 1988} & \multicolumn{2}{c|}{ Schmidt plates 1990} & \multicolumn{2}{c|}{ Schmidt plates 1994} \\
\hline Magnitude & $\sigma_{\alpha}(\mathrm{s})$ & $\sigma_{\delta}\left({ }^{\prime \prime}\right)$ & img. & $\sigma_{\alpha}(\mathrm{s})$ & $\sigma_{\delta}\left({ }^{\prime \prime}\right)$ & img. & $\sigma_{\alpha}(\mathrm{s})$ & $\sigma_{\delta}\left({ }^{\prime \prime}\right)$ & img. \\
\hline 4 & 0.003 & 0.11 & 14 & 0.026 & 0.24 & 27 & & & \\
5 & 0.004 & 0.08 & 31 & 0.020 & 0.39 & 35 & 0.012 & 0.13 & 116 \\
6 & 0.019 & 0.17 & 102 & 0.022 & 0.37 & 150 & 0.011 & 0.13 & 163 \\
7 & 0.007 & 0.10 & 102 & 0.017 & 0.28 & 173 & 0.014 & 0.13 & 285 \\
8 & 0.013 & 0.20 & 285 & 0.017 & 0.28 & 388 & 0.013 & 0.15 & 582 \\
9 & 0.013 & 0.19 & 384 & 0.017 & 0.28 & 644 & 0.011 & 0.12 & 1520 \\
10 & 0.011 & 0.14 & 866 & 0.017 & 0.28 & 1543 & 0.010 & 0.13 & 2829 \\
11 & 0.008 & 0.12 & 1471 & 0.017 & 0.30 & 3855 & 0.011 & 0.13 & 4679 \\
12 & 0.009 & 0.12 & 3216 & 0.017 & 0.29 & 4734 & 0.014 & 0.18 & 5807 \\
13 & 0.010 & 0.16 & 1714 & 0.018 & 0.28 & 4749 & 0.014 & 0.17 & 7091 \\
14 & 0.009 & 0.11 & 29 & 0.020 & 0.30 & 3061 & 0.017 & 0.21 & 5144 \\
15 & & & & 0.017 & 0.32 & 10 & 0.023 & 0.28 & 1560 \\
16 & & & & & & & 0.035 & 0.48 & 374 \\
\hline total errors & 0.010 & 0.14 & 8214 & 0.018 & 0.29 & 19372 & 0.015 & 0.18 & $30150 \mid$ \\
\hline *'s Hipp. & 0.004 & 0.02 & 138 & 0.005 & 0.12 & 140 & 0.008 & 0.17 & 133 \\
\hline
\end{tabular}




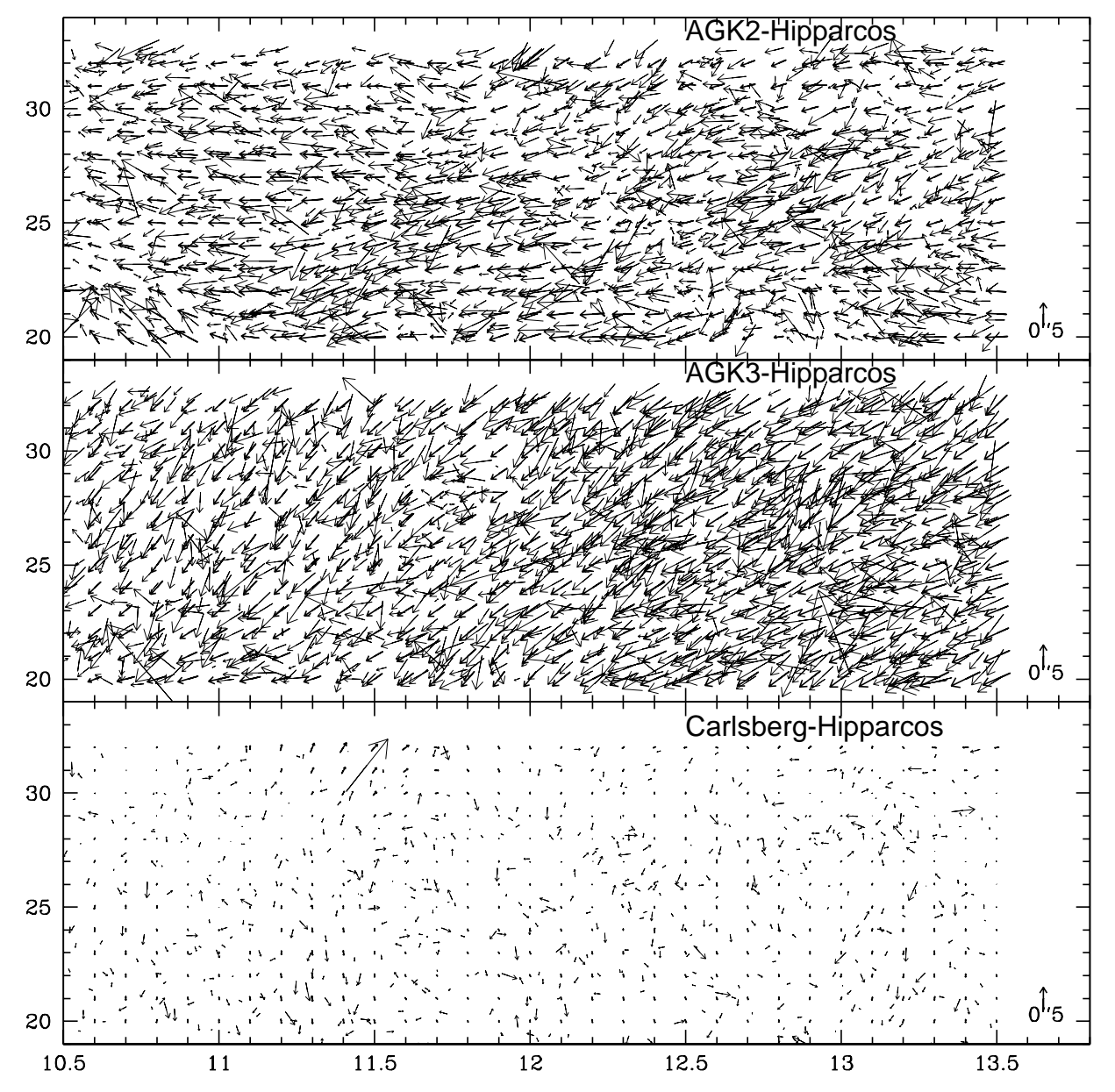

Fig. 1. Vector fields representing the positions differences between specific catalogues and the Hipparcos Catalogue. Light arrows represent individual differences. Each arrow has its origin on the point of coordinates $(\alpha, \delta)$ of the respective common star. Modulus and direction are given by the composition of the two $\alpha$ and $\delta$ differences. Hipparcos system and epoch of the catalogue to study are used. The heavy arrows represent the systematic difference obtained after adjustment of a sliding polynomial to real differences (see text). The AGK2, the AGK3, and the Carlsberg catalogues are represented in a separating form

derived from these scans by J. Lee of the Yale University using programs developed by himself. The original scans of this series were used to test an image adjustment method which was subsequently applied to the scans of the POSS plates. The POSS plates were digitized using the PDS microdensitometer of the Space Telescope Institute, and the data were made available to us. The plates of the series Schmidt 90 were measured with the Zeiss PSK2 stereocomparator of CIDA. For the Paris and Oxford zones of the Astrographic Catalogue and for the plate series by Heckmann (1929) the original measurements were used.

Each of the Schmidt plate series presented different specific problems. Systematic errors caused by the plate bending mechanism of the Schmidt Camera were found in Schmidt 88 series (Abad 1995). A small movement of the mirror of the telescope mainly on the $y$-axis direction was present when the Schmidt 90 plates were taken. And finally, light came into the box containing the Schmidt 94 plates, before they were taken, producing a small degradation of the back ground over one side of each plate.

Such problems as well as the saturation of the brightest stars of the POSS plates, were used to check a new function which reproduce the real profile of the digitalized images better than the gaussian functions. The function created make use of the arctangent function (Abad 1996).

$$
\begin{aligned}
& d(u, v)=\frac{A}{4 a \operatorname{tn}\left(b_{u} c_{u}\right) \operatorname{atn}\left(b_{v} c_{v}\right)} \\
& \left(\operatorname{atn}\left(b_{u}\left(u-c_{u}\right)\right)-\operatorname{atn}\left(b_{u}\left(u+c_{u}\right)\right)\right) \\
& \left(\operatorname{atn}\left(b_{v}\left(v-c_{v}\right)\right)-\operatorname{atn}\left(b_{v}\left(v+c_{v}\right)\right)\right)+\phi_{1}+\phi_{2} u+\phi_{3} v
\end{aligned}
$$




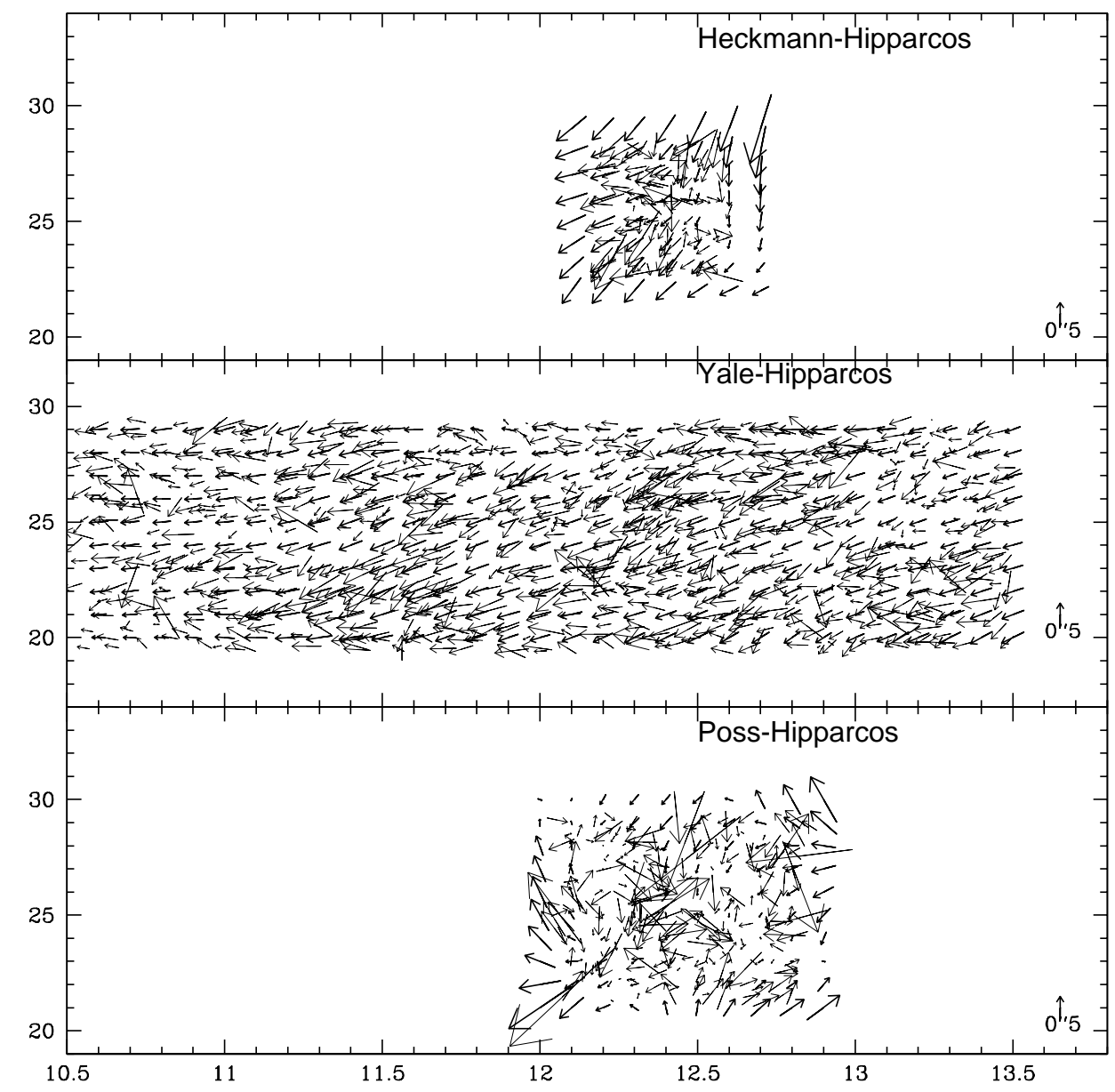

Fig. 2. Vector fields representing the positions differences between specific catalogues and the Hipparcos Catalogue. Light arrows represent individual differences. Each arrow has its origin on the point of coordinates $(\alpha, \delta)$ of the respective common star. Modulus and direction are given by the composition of the two $\alpha$ and $\delta$ differences. Hipparcos system and epoch of the catalogue to study are used. The heavy arrows represent the systematic difference obtained after adjustment of a sliding polynomial to real differences (see text). The Heckmann, the Yale and the POSS catalogues are represented in a separating form

Table 4. Proper motion error analysis of the catalogue

\begin{tabular}{|c|r|c|c|c|}
\hline Catalogues & Stars & $\alpha$ mean error & $\delta$ mean error & mean epoch differences \\
\hline$n^{0}$ & $n^{0}$ & $\sigma_{\alpha}$ (s/year) & $\sigma_{\delta}\left({ }^{\prime \prime} /\right.$ year $)$ & years \\
\hline$\geq 4$ & 2410 & 0.00019 & 0.0028 & 85.45 \\
$\geq 5$ & 1674 & 0.00012 & 0.0014 & 92.86 \\
$\geq 6$ & 638 & 0.00006 & 0.0008 & 94.78 \\
$\geq 7$ & 371 & 0.00005 & 0.0005 & 94.02 \\
$\geq 8$ & 265 & 0.00004 & 0.0004 & 93.92 \\
$\geq 9$ & 196 & 0.00003 & 0.0003 & 94.25 \\
$\geq 10$ & 138 & 0.00003 & 0.0003 & 94.31 \\
$\geq 11$ & 62 & 0.00003 & 0.0003 & 94.63 \\
$\geq 12$ & 7 & 0.00002 & 0.0003 & 100.11 \\
\hline
\end{tabular}




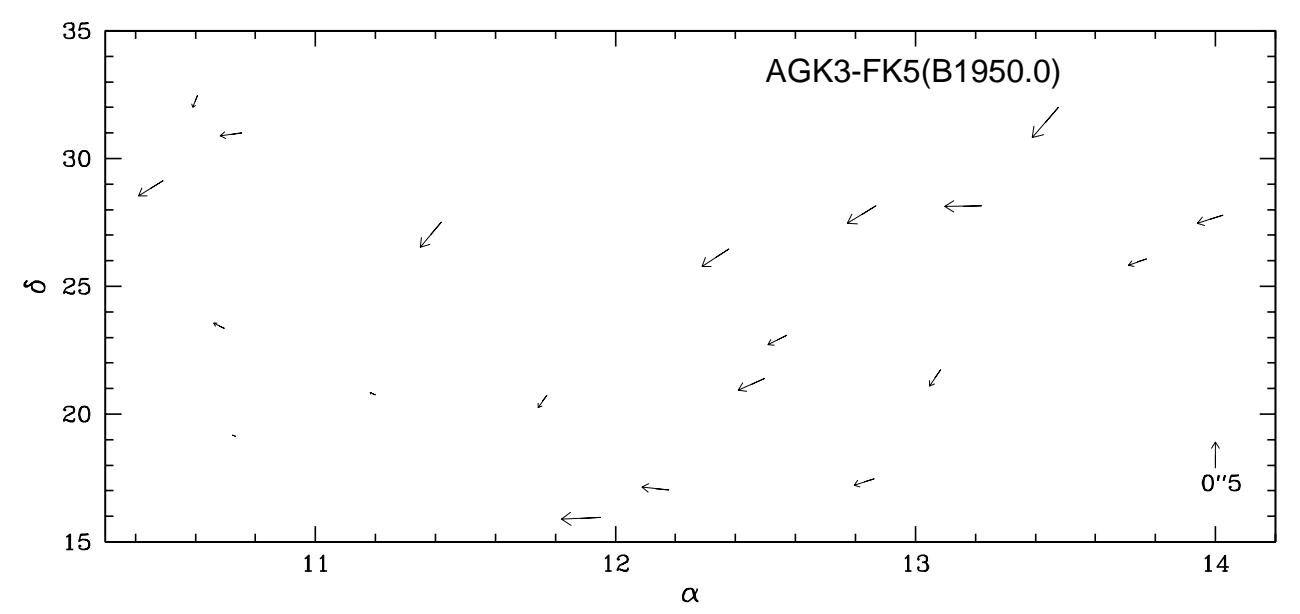

Fig. 3. Position differences between the AGK3 and the FK5 catalogs. For the FK5 positions the B1950.0 version was used, and corrected to the epoch of the AKG3 plates, using the proper motions of the FK5 catalog

where,

$u=\left(x-x_{0}\right) \cos (\phi)+\left(y-y_{0}\right) \sin (\phi)$

$v=-\left(x-x_{0}\right) \sin (\phi)+\left(y-y_{0}\right) \cos (\phi)$

$\left(x_{0}, y_{0}\right)$ center of the image,

$\phi$ angle between axis of symmetry of the image and axis of the digitalization,

$A$ maximum density of the image,

$b_{u}$ and $b_{v}$ slope parameters,

$c_{u}$ and $c_{v}$ width parameters and,

$\phi_{1}, \phi_{2}, \phi_{3}$ coefficients representing the background.

A simple modification of the above equation would make it possible to adapt the artificially created profile to the asymmetric real profile. Varying $b_{u}, b_{v}, c_{u}$ and $c_{v}$ different symmetries can be produced in either axis, thus permitting adaptation to a large variety of image shapes. The above profile is particularly useful for images with a saturated center and hence a flat-topped profile. The latter is the case for at least most of the reference stars. In fact, for saturated images the above profile equation is superior to the gaussian approximation, while for faint images the latter gives more consistent results. The parameters of the function, above all the coordinates of the center, are determined by least squares in an iterative process. The profile parameters are strongly related to the image brightness, which is related to the total pixel count. Thus initial values for all parameters can readily be found once the mentioned relations are approximately known.

In this paper only images of the POSS plates were analyzed with the new function.

In order to get the equatorial coordinates a linear block adjustment method derived by Stock (1981) was used for each plate series. After this first step, the plate model explained and applied by Abad (1998) is used. It consists in obtaining the field deformation depending on the position on the plate and on the magnitude from the differences, star by star, between the individual plate position and their averaged final position or the reference catalogue position if the star is contained in the reference catalogue. The individual plate position and their averaged final position were calculated both from the linear block adjustment. From these differences a general plate distortion pattern is derived which is then applied to the measured coordinates. A new block adjustment is carried out with the corrected coordinates, and these are again analyzed for a systematic distortion pattern. This process can be repeated until no systematic pattern is evident. Tables 2 and 3 contain the final results, separated into magnitude intervals for everyone of the series of plates or catalogs.

The catalogs which give their data in equatorial coordinates (Yale, Carlsberg, AGK2, AGK3, and the POSS data which were reduced with the PPM catalog as reference system) were all reduced to the Hipparcos system applying a weighted sliding polynomial scheme described by Stock \& Abad (1988). The process interpolates the differences between the Hipparcos positions and the positions given by the respective catalog for stars common to both, making use only of stars up to a given maximum distance from the star whose position is to be corrected.

In order to convert the equinox and orientation of the catalog data to coincide to Hipparcos system, expressions given by Fricke et al. (1988) are used. An inadequate precession constants will simply add to the systematic differences which have to be determined anyway. To the position of the Hipparcos catalog the respective proper motions were added to allow for the epoch differences between Hipparcos and the catalog.

Weights are assigned to the differences, based on the same distances in the sense that the weight reaches unity at the point to be interpolated, and zero at the maximum distance. Using this interpolation scheme at equidistant coordinate intervals the distortion pattern of the respective catalog can be found. Example are shown in Figs. 1 and 2. Systematic differences, at times surprisingly large, are found between the AGK2 and AGK3 catalogs and the 
Hipparcos catalog. In order to show that these differences are real we shall compare as an example the difference between the AGK3 and the FK5, using the B1950.0 version of the latter. The coordinates of the AKG3 are given in the same system. Thus only the epoch difference of around nine years has to be taken into account. Proper motions corrections were applied to the positions of the FK5. The resulting differences are shown as an arrow diagram in Fig. 3. It may be noted that the pattern in this figure is quite similar to that in Fig. 1 in the case of the AGK3.

\section{The catalog}

The final catalog (Table 5) contains positions and proper motions for approximately 2400 stars which have been selected with the following criteria: (1) the star must appear in at least 4 of the contributing catalogs, and (2) the maximum epoch difference must be at least 40 years. A photographic magnitude is also given for every object. In the case of the photographic sources the image sizes or total pixel counts were calibrated using the magnitudes in the PPM catalog, and extrapolated to fainter magnitudes. Spectral types are also given, taken from any of the sources. For the determination of the final data weights are given to each of the contributing catalogs according to the average respective mean errors. The contents of the catalogue are as follows:

$1(N)$ running number of this catalog,

2 ( $m$ ) estimated photographic magnitude,

3 (st) spectral type,

4 ( $\alpha$ ) right ascension (hours),

$5(\alpha)$ right ascension (minutes),

6 ( $\alpha)$ right ascension (seconds),

$7\left(\sigma_{\alpha}\right)$ mean error of right ascension (seconds of time $\times 0.001)$,

$8\left(\mu_{\alpha}\right)$ proper motion in right ascension (seconds of time/year),

$9 \quad\left(\sigma_{\mu_{\alpha}}\right)$ error of proper motion in right ascension (seconds of time $\times 0.00001 /$ year $)$,

$10(\delta)$ declination (degrees),

$11(\delta)$ declination (arcminutes),

$12(\delta)$ declination (arcseconds),

$13\left(\sigma_{\delta}\right)$ mean error of declination (arcseconds $\times 0.01$ ),

$14\left(t_{\mathrm{m}}\right)$ epoch of right ascension and declination,

$15\left(\mu_{\delta}\right)$ proper motion in declination (arcseconds/year),

$16\left(\sigma_{\mu_{\delta}}\right)$ mean error of proper motion in declination (arcseconds $\times 0.0001 /$ year),

$17(\Delta T)$ maximum epoch difference,

$18(C)$ number of contributing catalogs.

The average mean errors of the proper motions, both in right ascension and declination, are $0.003 \mathrm{arcsec} /$ year if there are at least 4 contributors, going down to 0.0005 arcsec/year if there are 7 or more contributors. A proper motions error analysis is given in Table 4. A comparison of our catalog with the Hipparcos data, both positions and proper motions, show no systematic pattern. Thus our catalog can be considered to be on the system defined by Hipparcos.

Acknowledgements. The POSS data used in this work are based on plates of the National Geographic Society Palomar Observatory Sky Survey (NGS-POSS) obtained with the Oschim Telescope of the Mount Palomar Observatory. The NGS-POSS is funded by the National Geographic Society of the California Institute of Technology. The Digitized Sky Survey was carried out at the Space Telescope Science Institute, Baltimore, funded by the National Science Foundation under the contract NAC W-2166. We also thank J. Stock for his collaboration during the elaboration of this paper.

\section{References}

Abad C., 1993, A\&AS 98, 1

Abad C., 1995, A\&AS 111, 369

Abad C., 1996, Ph. D. thesis, Publicaciones del Seminario Matemático García de Galdeano, Serie II, No. 52 Universidad de Zaragoza, Zaragoza, Spain

Abad C., 1998, A\&AS 132, 275

AGK2 Star Catalogue of Positions and Proper Motions North of $-2^{\circ} 5$ Declination, Derived from Photographic Plates taken at Bergedorf and Bonn in the years 1928-1932

AGK3 Star Catalogue of Positions and Proper Motions North of $-2^{\circ} 5$ Declination, Derived from Photographic Plates taken at Bergedorf and Bonn in the years 1928-1932 and 1956-1963

Boss B., 1936, General Catalogue of 33342 Stars for the Epoch 1950, Vols. III \& IV, Carnegie Institution of Washington, Washington 1936

Carlsberg Meridian Catalogue La Palma No. 9: Observations of positions of stars and planets: May 1984 to March 1995, including Extinction and Meterealogical data (CMC9) 1997, Copenhagen University Observatory, Royal Greenwich Observatory and Real Instituto y Observatorio de la Armada en San Fernando

Heckmann O., 1929, Veroff. Univ. Sternwarte Gottingen 1, 141

Fricke W, Schwan H., Lederle T., 1988, Fifth Fundamental Catalogue (FK5), Part I, The Basic Fundamental Stars, A Publ. of the Astronomisches Rechen-Institute Heidelberg

Roeser S., Bastian U., 1991, PPM Star Catalogue. Positions and Proper Motions of 181731 stars north of -2.5 degrees declination for equinox and epoch J2000.0. A Publ. of the Astronomisches Rechen-Institute Heidelberg

Stock J., 1981, Rev. Mex. Astron. Astrofis. 6, 115

Stock J., Abad C., 1988, Rev. Mex. Astron. Astrofis. 16, 63

Trumpler R.J., 1938, Lick Obs. Bull. 18, 167

Yale Catalogue, Transactions of the Astronomical Observatory of Yale University, Vol. 24, Revised Catalogue of 10358 stars, +25 to +30 by Ida Barney. New Haven, Published by the Observatory, 1953

Yale Catalogue. Transactions of the Astronomical Observatory of Yale University, Vol. 25, Revised Catalogue of 8703 stars, +20 to +25 by Ida Barney. New Haven, Published by the Observatory, 1954 